\title{
The intriguing properties of local compact massive galaxies: What are they?
}

\author{
A. Ferré-Mateu ${ }^{1,2}$, A. Vazdekis ${ }^{1,2}$, I. Trujillo ${ }^{1,2}$, P. Sánchez-Blázquez ${ }^{3}$, \\ E. Ricciardelli ${ }^{4}$ and I. G. de la Rosa ${ }^{1,2}$ \\ ${ }^{1}$ Instituto de Astrofísica de Canarias, La Laguna, SPAIN \\ email: aferre@iac.es \\ ${ }^{2}$ Departamento de Astrofísica, Universidad de La Laguna, SPAIN \\ ${ }^{3}$ Departamento de Física Teórica, Univ. Autónoma de Madrid, Madrid, SPAIN \\ ${ }^{4}$ Departament d'Astronomia i Astrofísica, Universitat de València, SPAIN
}

\begin{abstract}
Studying the properties of the few compact massive galaxies that exist in the local Universe (Trujillo et al. 2009) might provide a closer look to the nature of their high redshift $(\mathrm{z} \geqslant 1.0)$ massive counterparts. By this means we have characterized their main kinematics, structural properties, stellar populations and star formation histories with a set of new high quality spectroscopic and imaging data (Ferré-Mateu et al. 2012 and Trujillo et al. 2012). These galaxies seem to be truly unique, as they do not follow the characteristic kinematics, stellar surface mass density profiles and stellar population patterns of present-day massive ellipticals or spirals of similar mass. They are, instead, more alike their high-z analogs.

Summarizing, local compact massive galaxies are rare, unique and the perfect laboratory to study their high redshift counterparts.
\end{abstract}

Keywords. galaxies: evolution, galaxies: formation, galaxies: fundamental parameters, galaxies: kinematics and dynamics, galaxies: stellar content
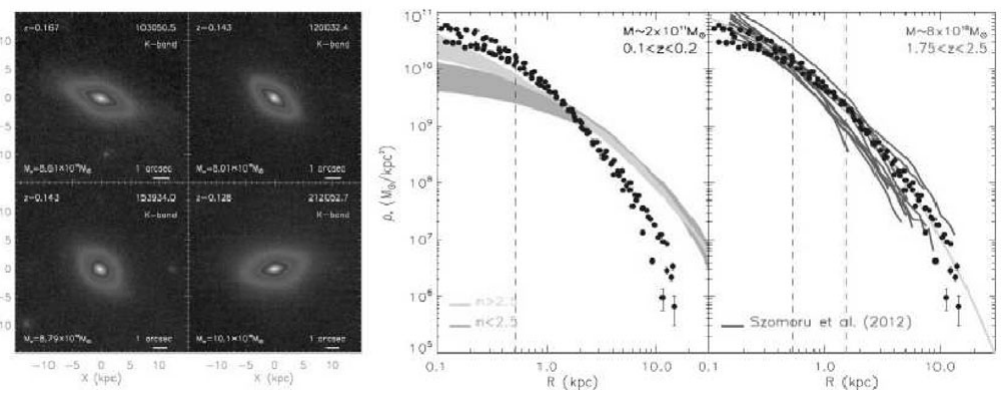

Figure 1. Left K-band high resolution imaging of four nearby massive compact galaxies with galaxy stellar mass, spectroscopic redshift, and the equivalent $1^{\prime \prime}$ angular size (solid line). Right Stellar surface mass density profiles of the local compact massive galaxies (black points) (i) compared with the stellar mass density profiles of SDSS DR7 $\mathrm{M}_{*} \geqslant 2 \times 10^{11} M_{\text {sun }}$ and $0.1<\mathrm{z}$ $<0.2$ disk-like galaxies $(\mathrm{n}<2.5)$ and spheroid-like $(\mathrm{n}>2.5)$ galaxies. (ii) compared with $\mathrm{z} \sim 2$ massive compact galaxies of same stellar mass (Szomoru et al. 2012).

\section{References}

Ferré-Mateu, A., et al. 2012, MNRAS, 423, 632

Trujillo, I., et al. 2009, ApJ, 692, 118

Trujillo, I., Carrasco, R. E., \& Ferré-Mateu, A. 2012, ApJ, 751,45

Szomoru, D., et al. 2012, ApJ, 749, 121 